\title{
Detecting dengue outbreaks in Malaysia using geospatial techniques
}

\author{
Syahrul Nellis, ${ }^{1}$ Shih Keng Loong, ${ }^{2}$ Juraina Abd-Jamil, ${ }^{2}$ Rosmadi Fauzi, ${ }^{3}$ Sazaly AbuBakar ${ }^{1,2}$ \\ ${ }^{1}$ Department of Medical Microbiology, Faculty of Medicine, Universiti Malaya; ${ }^{2}$ Tropical Infectious \\ Diseases Research \& Education Centre (TIDREC), Universiti Malaya; ${ }^{3}$ Department of Geography, Faculty \\ of Arts and Social Sciences, Universiti Malaya, Kuala Lumpur, Malaysia
}

\begin{abstract}
Dengue is a complex disease with an increasing number of infections worldwide. This study aimed to analyse spatiotemporal dengue outbreaks using geospatial techniques and examine the effects of the weather on dengue outbreaks in the Klang Valley area, Kuala Lumpur, Malaysia. Daily weather variables including rainfall, temperature (maximum and minimum) and wind speed were acquired together with the daily reported dengue cases data from 2001 to 2011 and converted into geospatial format to identify whether there was a specific pattern of the dengue outbreaks. The association between these variables and dengue outbreaks was assessed using Spearman's correlation. The result showed that dengue outbreaks consistently occurred in the study area during a 11-year study period. And that the strongest outbreaks frequently occurred in two high-rise apartment buildings located in Kuala Lumpur City centre. The results also show significant negative correlations between maximum temperature and minimum temperature on dengue outbreaks around the study area as well as in the area of the high-rise apartment buildings in Kuala Lumpur City centre.
\end{abstract}

Correspondence: Sazaly AbuBakar, Tropical Infectious Diseases Research \& Education Centre, Universiti Malaya, 50603 Kuala Lumpur, Malaysia. E-mail: sazaly@um.edu.my

Key words: Geography information system; spatial analysis; health geography; epidemiology study; dengue study; Malaysia.

Funding: we acknowledge the funding from the Ministry of Higher Education, Malaysia for niche area research under the Higher Institution Centre of Excellence program (MO002-2019), the University Malaya Postgraduate Research Fund (PF126-2012B) \& support from the Ministry of Higher Education, Malaysia MyBrain15 scholarship.

Conflict of interest: the authors declare no potential conflict of interest.

Received for publication: 25 January 2021.

Revision received: 10 June 2021.

Accepted for publication: 18 June 2021.

(C) Copyright: the Author(s), 2021

Licensee PAGEPress, Italy

Geospatial Health 2021; 16:1008

doi:10.4081/gh.2021.1008

This article is distributed under the terms of the Creative Commons Attribution Noncommercial License (CC BY-NC 4.0) which permits any noncommercial use, distribution, and reproduction in any medium, provided the original author(s) and source are credited.

\section{Introduction}

Dengue fever (DF) is a vector-borne disease caused by the dengue virus (DENV) transmitted by the mosquito species, Aedes aegypti and Ae. albopictus (Christie, 1881; Senthil et al., 2014; Shaikh et al., 2014). Ae. aegypti is the primary vector of DF in tropical and subtropical regions. Both mosquito species breed in rural and suburban areas (Hawley et al., 1987; Rodhain et al., 1997). After it has been transmitted to a person from the bite of a female mosquito, DENV produces a viraemic state causing the infected person to develop mild to severe DF. Mild infection resembles an influenza-like illness, whereas a severe DF can advance to dengue haemorrhagic fever (DHF) and dengue shock syndrome (DSS) (Gibbons, 2010; Guzman and Istúriz, 2010; Gubler, 2011).

DF incidence has increased globally, particularly affecting those living in tropical and subtropical regions in Southeast Asia, the Americas, and Asia Pacific (Halstead, 2007; Simmons et al., 2012; Yasuno and Tonn, 1970). Approximately 2.5 billion people in 120 countries are at risk of dengue infection $(\mathrm{Ng}, 2011$; Otu et al., 2019) and more than 50 million dengue infections are reported each year, with more than 500,000 individuals hospitalised due to the DF (Guzman et al., 2010). The number of reported DF cases increases each year, causing high mortality globally (Beatty et al., 2011).

Since the $20^{\text {th }}$ century, DF has become the most critical public health issue in urban areas due to the increase in urbanisation, globalisation, lack of mosquito control strategies and climate change. The urban areas provide an ideal breeding environment for the Aedes mosquitoes. Several species breed in artificial human-made environments, such as illegal dumpsites and building sites (Chang et al., 2011). Moreover, stagnant water trapped in non-biodegradable containers, such as vehicle tyres, plant pots and the like during the rainy season becomes the breeding ground for these mosquitoes (Lowe et al., 2011; Wan-Norafikah et al., 2012). Collectively, the factors mentioned above contribute to DENV transmission.

In Malaysia, the DF endemic is a never-ending war since Penang's first dengue case in 1902 (Skae, 1902). Ever since then, dengue has become a significant public health problem in the country (Smith, 1957; Rudnick et al., 1965). Although many initiatives based on legislation, community participation, pesticides (either active against the adult mosquitoes or the larvae) and integrated control strategies have been taken, the number of reported dengue cases continues to rise (Lee et al., 2008; Malaysia, 2009; Nazni et al., 2009). In Malaysia, the cumulative dengue case was $157 \%$ higher in 2019 compared to 2018 (World Health Organisation, 2019). By the end of July 2019, Malaysia reported 79,151 dengue cases nationwide, with the Selangor state contributing 40,849 (Crisis Preparedness and Response Centre, 
2020). The increasing number of dengue cases is alarming for a developing country like Malaysia because it has highly populated city centres (e.g. Petaling Jaya and Kuala Lumpur) suitable for dengue transmission. Some studies done in Malaysia argue that the presence of Aedes mosquitoes and increasing dengue cases may be associated with the dense urban population (Nazri et al., 2009, World Health Organization, 2009).

Geographic information systems (GIS), a robust approach to geospatial analysis, have been applied in epidemiological and public health studies for many years assessing dengue location and identifying potential risk factors involved in disease transmission (Mohammed et al., 2019; Moha et al., 2020; Murugesan et al., 2020). To date, GIS dengue applications in Malaysia have only used the individual dengue case data and focused on small local areas of Klang Valley near the nation's capital, Kuala Lumpur (e.g. Subang Jaya and Hulu Langat districts in Selangor) (Nazri et al., 2009; Dom et al., 2010; Er et al., 2010). However, there is still a lack of evidence with regard to outbreak identification and the area of vulnerability in the Klang Valley area. This research's primary aim was to identify the dengue outbreak location together with analysing the spatiotemporal trend of dengue outbreaks using geospatial techniques in Malaysia's most urbanised area.

\section{Materials and methods}

\section{Study area}

Kuala Lumpur and Petaling Jaya are in the heart of Klang Valley, one of Malaysia's fastest growing urban areas (Figure 1). The Klang Valley is the centre of Malaysia's industry and commerce. The latest census report indicates that this area's population has expanded to 6.3 million (SPAD, 2016). Spatial data regarding the administrative boundaries (state, district, sub-district, boundary map, road map, Klang Valley city centre and local authority boundary map) were collected from the Klang Valley local authorities and the Department of Survey and Mapping Malaysia (JUPEM).

The Petaling District, which includes Petaling Jaya, Shah Alam, and Subang Jaya have recorded the highest number of dengue cases in Selangor (JKN Selangor, 2016). From the University Malaya Medical Centre (UMMC), we obtained the confirmed dengue case data for its catchment area $\left(101^{\circ} 39^{\prime} 11.50^{\prime \prime} \mathrm{E}\right.$, $3^{\circ} 6^{\prime} 48.59^{\prime \prime N}$ ) (Figure 1). This hospital was chosen for this study because it is situated in the Petaling District and Kuala Lumpur, with a consistently high number of dengue cases. Furthermore, UMMC maintains an annual record of dengue cases required in

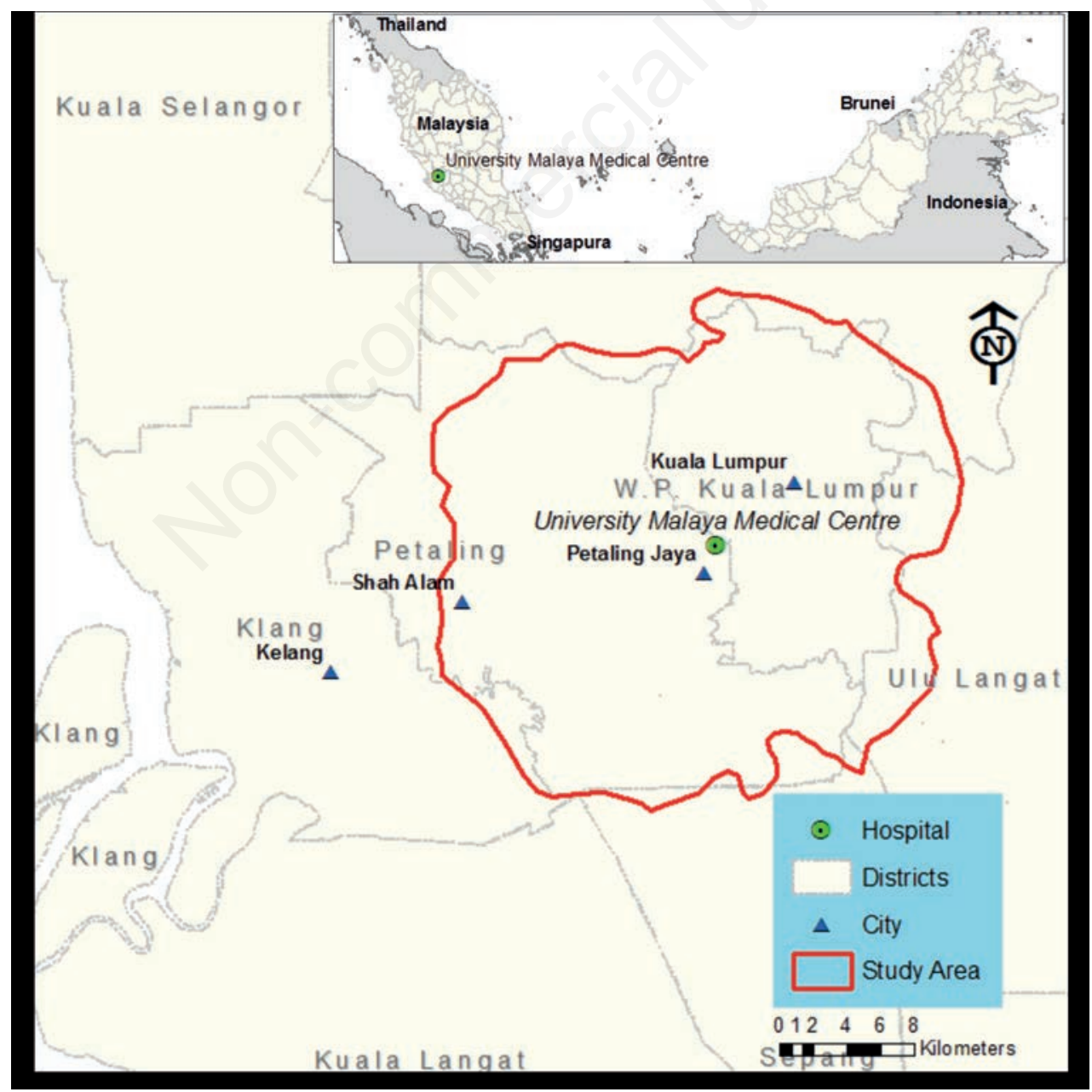

Figure 1. Study area location. 
understanding the characteristics of dengue transmission in Malaysia.

\section{Data acquisition}

\section{Dengue cases}

These data were obtained from the UMMC. Only dengue cases confirmed through laboratory testing using serological tests for immunoglobulin $\mathrm{M}(\operatorname{IgM})$ antibody against any of the four different dengue serotypes (IgM capture enzyme-linked immunosorbent assay; ELISA) were included in this study. The reason for using laboratory-confirmed data rather than the reported dengue cases was to avoid inaccurate information and to know which cases were new. Cases could have been misdiagnosed since signs and symptoms of other diseases, such as typhoid and malaria are similar to those of dengue. In addition, the reported cases may not include all those infected since it does not include asymptomatic patients. We also acquired age, gender and geographical address of the confirmed dengue cases as well as information and photographs of known dengue outbreak locations from the Department of Health and Environment of Kuala Lumpur City Hall (DBKL).

\section{Meteorological data}

Daily meteorological data for the period of January 2001 until December 2011 were obtained from Meteorological Department, Malaysia. The variables used to examine the effects of the weather on the dengue outbreak pattern included rainfall, maximum and minimum temperature, wind speed, and humidity. Spearman's correlation coefficient $(r)$ was used to determine the relationship between various variables. This method provides accurate information about the variables' relationships, which is crucial. The results vary between +1 and -1 , where the positive correlation $(r=$ $+1)$ refers to a $45^{\circ}$ upward line slope, while the negative $(r=-1)$ refers a $45^{\circ}$ downward line slope. A positive $r$ value $($ near +1$)$ indicates that the increase of weather variables would also increase the dengue outbreak, while a negative $r$ value $($ near -1$)$ indicates that the increase of weather variables will reduce the dengue outbreak. Values of $r$ around 0 mean a random relationship. All statistical analyses were performed using the Statistical Package for the Social Sciences (SPSS) software.

\section{Geospatial and statistical analysis}

First, point level geo-coding technique was applied by converting the patients' addresses into an absolute location (latitude and longitude) using Google Earth. The absolute location data first was standardised using Microsoft Excel. Next, the standardised data were imported to ArcGIS software version 10.5. There were 9348 dengue cases throughout the study area in the 2001-2011 period.

To pinpoint the location of the dengue outbreak, we applied quadrant analysis, which involved superimposing previous dengue cases data onto a regular square grid. In line with the Malaysian Ministry of Health's official definition of a dengue outbreak (i.e. the occurrence of two or more dengue cases $\leq 200 \mathrm{~m}$ of each other and less than 14 days apart as described by the Malaysian Remote Sensing Agency (2014)), we distinguished between 1 (=two or more dengue cases) and 0 (=one no dengue case) on a systematic 200-meter grid of projected coordinates Kertau RSO Malaya (m) system for the study area. The Kertau RSO Malaya meter was the common map projection that has been in topographic mapping and engineering surveys for Malaysia. The triangulated irregular network (TIN) method (Academic Press, 2020) was applied to the final data in ArcGIS to create a three-dimensional (3D) mapping overlaying of the 11-year results.

\section{Results}

A total of 1274 outbreaks were identified from 2001 until 2011 (Table 1). The highest number of reported outbreaks was 226 occurred in 2008 followed by 195 in 2004 and 174 in 2007 . We found that the dengue outbreaks had 2-cycle patterns, with the first starting in 2001when the number of outbreaks continued to increase until it peaked in 2004 and then dropped drastically in 2005. The second cycle started in 2006 demonstrating the same outline as the first one. Here the number of outbreaks continued to increase until peaking in 2008 and then dropping to its lowest in 2011. This retrospective study indicated that dengue outbreaks had a pattern with cycles that occurred continuously for an average of two consecutive years

The dengue outbreak map generated from the quadrant analysis is shown in Figure 2 where the outbreaks are colour-coded, with the lowest number of outbreaks in dark green and the highest in red. Most outbreaks were centred at the Kuala Lumpur area, in particular around the west side of Kuala Lumpur city, which is close to the Petaling Jaya border and known as Zone 1. In addition, outbreak locations were also detected around the western area of Petaling Jaya. As further seen in Figure 2, there are several areas in Kuala Lumpur and Petaling Jaya that consistently score the highest number of dengue outbreaks. For example, Zone 1 in Kuala Lumpur not only recorded the highest number of outbreaks in 2004 (76) but also the highest dengue outbreak number for five more years: 2002 (29), 2003 (27), 2006 (31), 2007 (25) and 2009 (17). A change of outbreak distribution was noted in 2006, when the higher concentration of dengue outbreaks switched from western Kuala Lumpur to the western part of Petaling Jaya.

The highest dengue outbreaks persistently occurred in residential areas (Figure 3). The analysis showed that there are two highrise apartment buildings located in Zone 1 of Kuala Lumpur city centre in the area where the highest dengue outbreak number was consistently recorded. Apartment Building 1 is situated in Zone 1,

Table 1. The annual number of dengue outbreaks between 2001 and 2011.

\begin{tabular}{lc} 
Tlime (year) & Outbreak (no.) \\
2001 & 43 \\
2002 & 85 \\
\hline 2003 & 87 \\
2004 & 195 \\
2005 & 52 \\
\hline 2006 & 65 \\
2007 & 174 \\
\hline 2008 & 226 \\
2009 & 156 \\
\hline 2010 & 117 \\
2011 & 74 \\
\hline Total & 1274 \\
\hline
\end{tabular}



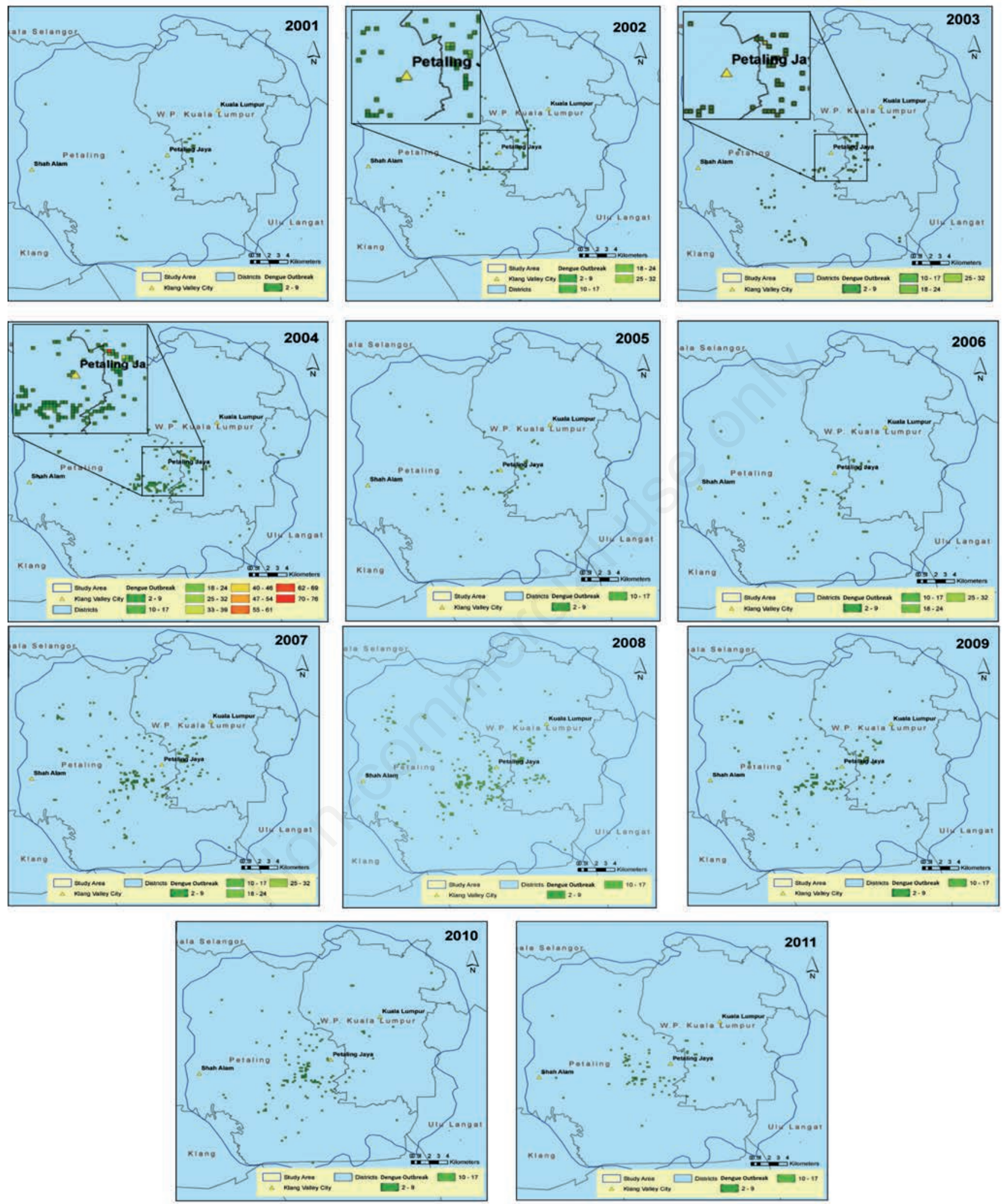

Figure 2. Distribution and number of annual dengue outbreak locations from 2001 to 2011 . The highest number of outbreaks occurred in 2003 (27), 2002 (29) and 2004 (76). 
where the consistently high number of outbreaks had been recorded. A similar result was also observed in Petaling Jaya, which saw the highest dengue outbreak number in 2008 (13) followed by 2010 (13) and 2011 (17).

Figure 4 shows the photographs of the two high-rise, residential apartment buildings, both with over ten floors. These two buildings reported the highest dengue outbreaks in Kuala Lumpur during the study period. Figure 5 shows stagnant water in the drain system of Apartment Building 1 and 2 (photograph provided by the DBKL).

The results of Spearman's correlation analysis of the environmental variables with respect to dengue outbreaks are displayed in Tables 2 and 3, the former dealing within the whole study area and the latter with Zone 1 in Kuala Lumpur. In both areas, the maximum temperature was found to negatively correlate with the dengue outbreaks at a statistically significant level, as did the minimum temperature but less strongly so.

We also found that some of the variables showed weak negative correlations, i.e. rainfall and temperature in the whole study area, as well as rainfall, temperature and humidity in Zone 1, while some others did not. However, the wind variables had a weak positive correlation in both areas.

\section{Discussion}

The primary aim of this study was to analyse the spatiotemporal trend of dengue outbreaks using geospatial techniques in Malaysia's most urbanised area. Our analysis revealed that the highest number of dengue outbreaks consistently occurred in residential areas, which was particularly pronounced at the vicinity of two high-rise apartment buildings. Like other urban cities in other developing countries, most of the population live in densely populated neighbourhoods (e.g. in apartment buildings). This situation results from rapid urban development and urban processes that affect the influx of people from rural areas to the city, resulting in local explosive population growth (Honório et al., 2009; Braga et al., 2010; Paz-Soldan et al., 2011). High living costs force people to find comparatively low-cost residential areas and their choice has often been apartment in buildings with ten floors or higher, e.g. Pantai Dalam in Kuala Lumpur and Old Klang road area in Petaling Jaya.

High-density populations in endemic areas increase dengue transmission that can lead to dengue outbreaks (Wen et al., 2010), a risk which is exacerbated by waste management failure. Other than that, residents' low awareness and attitudes, such as disposing of rubbish (e.g. non-biodegradable plastics, car tyres and tin cans) everywhere, work as trigger events (Hayes et al., 2003, Ma et al., 2008). Such conditions eventually become suitable habitats for mosquitoes, thereby increasing vector opportunities for Ae. aegypti and other carriers of the infection (Lawson et al., 2001) resulting in increased biting rates and infections (Er et al., 2010). Similar findings have also been reported in previous studies done in several Asia Pacific countries (Wen et al., 2006; Er et al., 2010; Wen et al., 2010).

The structure of residential buildings is another factor that may cause dengue outbreaks (Aris, 2019). Most dengue outbreaks found in this study occurred in people living in flats in high-rise apartment buildings. Iyagba (2005) and Cheong (2014) explained that building factors, especially related to high-rise buildings, can contribute to dengue outbreaks in urban areas (Iyagba, 2005; Cheong et al., 2014). Thus, building structures and conditions can become trigger factors, e.g. for the dengue outbreaks. This possi-

Table 2. Correlation between environmental variables and dengue outbreaks in the study area.

\begin{tabular}{lccccccccc} 
Variable & Rainfall & Temp & Hare & Max. temp. & Min. temp. & Wind & Humidity & Dengue \\
Rainfall & $\mathbf{1 . 0 0}$ & -0.10 & -0.26 & -0.04 & -0.05 & -0.02 & 0.12 & -0.04 \\
Temp. & -0.10 & $\mathbf{1 . 0 0}$ & 0.17 & 0.32 & 0.23 & -0.01 & 0.09 & -0.03 \\
\hline Haze & -0.26 & 0.17 & $\mathbf{1 . 0 0}$ & 0.17 & 0.05 & 0.03 & -0.12 & 0.00 \\
Max. temp. & -0.04 & 0.32 & 0.17 & $\mathbf{1 . 0 0}$ & 0.47 & 0.06 & -0.08 & $-0.21^{*}$ \\
\hline Min. temp. & -0.05 & 0.23 & 0.05 & 0.47 & $\mathbf{1 . 0 0}$ & 0.10 & 0.05 & $-0.14^{*}$ \\
Wind & -0.02 & -0.01 & 0.03 & 0.06 & 0.10 & $\mathbf{1 . 0 0}$ & -0.10 & 0.01 \\
Humidity & 0.12 & 0.09 & -0.12 & -0.08 & 0.05 & -0.10 & $\mathbf{1 . 0 0}$ & 0.01 \\
Dengue & -0.04 & -0.03 & 0.00 & $-0.21^{*}$ & $-0.14^{*}$ & 0.01 & 0.01 & $\mathbf{1 . 0 0}$ \\
\hline
\end{tabular}

*Significant correlation between dengue with environmental variables.

Table 3. Correlation between environmental variables and dengue outbreaks in Kuala Lumpur's Zone 1.

\begin{tabular}{lccccccccc} 
Variable & Rainfall & Temp & Haze & Max. temp. & Min. temp. & Wind & Humidity & Dengue \\
Rainfall & $\mathbf{1 . 0 0}$ & -0.10 & -0.26 & -0.04 & -0.05 & -0.02 & 0.12 & -0.05 \\
Temp. & -0.10 & $\mathbf{1 . 0 0}$ & 0.17 & 0.32 & 0.23 & -0.01 & 0.09 & -0.08 \\
\hline Haze & -0.26 & 0.17 & $\mathbf{1 . 0 0}$ & 0.17 & 0.05 & 0.03 & -0.12 & 0.03 \\
Max. temp. & -0.04 & 0.32 & 0.17 & $\mathbf{1 . 0 0}$ & 0.47 & 0.06 & -0.08 & $-0.27^{*}$ \\
\hline Min. temp. & -0.05 & 0.23 & 0.05 & 0.47 & $\mathbf{1 . 0 0}$ & 0.10 & 0.05 & $-0.21^{*}$ \\
Wind & -0.02 & -0.01 & 0.03 & 0.06 & 0.10 & $\mathbf{1 . 0 0}$ & -0.10 & 0.05 \\
\hline Humidity & 0.12 & 0.09 & -0.12 & -0.08 & 0.05 & -0.10 & $\mathbf{1 . 0 0}$ & -0.05 \\
Dengue & -0.05 & -0.08 & 0.03 & $-0.27^{*}$ & $-0.21^{*}$ & 0.05 & -0.05 & $\mathbf{1 . 0 0}$ \\
\hline
\end{tabular}

*Significant correlation between dengue with environmental variables. 
bility is plausible when examining the building gutters on the roof designed to remove rainwater. Notably, the gutters were not covered to prevent the accumulation of rubbish, leaves, debris, and dust. The accumulation of these objects clogged the gutter and caused the rainwater to stagnate, thus providing an ideal habitat for the mosquitoes to breed.

Other building elements, such as improper floor surfaces, also contribute to dengue upsurge by providing ideal conditions for Aedes mosquitoes to breed. Previous studies suggest that the building's structure and material can increase the Aedes populations, for example when debris accumulate on the floor causing water to be blocked and stagnated (Montgomery and Ritchie, 2002; Sairi et al., 2016). Other types of building structures (e.g. corridors and walkways) could also become breeding grounds for Aedes mosquitoes. Several studies in Asian countries show similar findings as our study (Thammapalo et al., 2005; Lau et al., 2013; Lee et al., 2013), which support the idea that high-rise apartment buildings provide a complete ecosystem for the Aedes mosquitoes. However, further research is warranted to investigate the influence of different building's structural design (e.g. terraces, balconies, gutters, roof structures and floor finishes) on Aedes reproduction. This study's findings could be a starting point for future dengue epidemiological study in Klang Valley. Health officials and policymakers could use these results to plan sustainable urban development policies.

This study's secondary aim was to investigate the effects of the weather on dengue outbreak frequency. Although the association between climate and dengue are complex and challenging to study, we found a negative association in relation to maximum and minimum temperatures in the locations studied. These findings are similar to those reported by $\mathrm{Yu}$ (2011) and Jemal and Al-Thukair

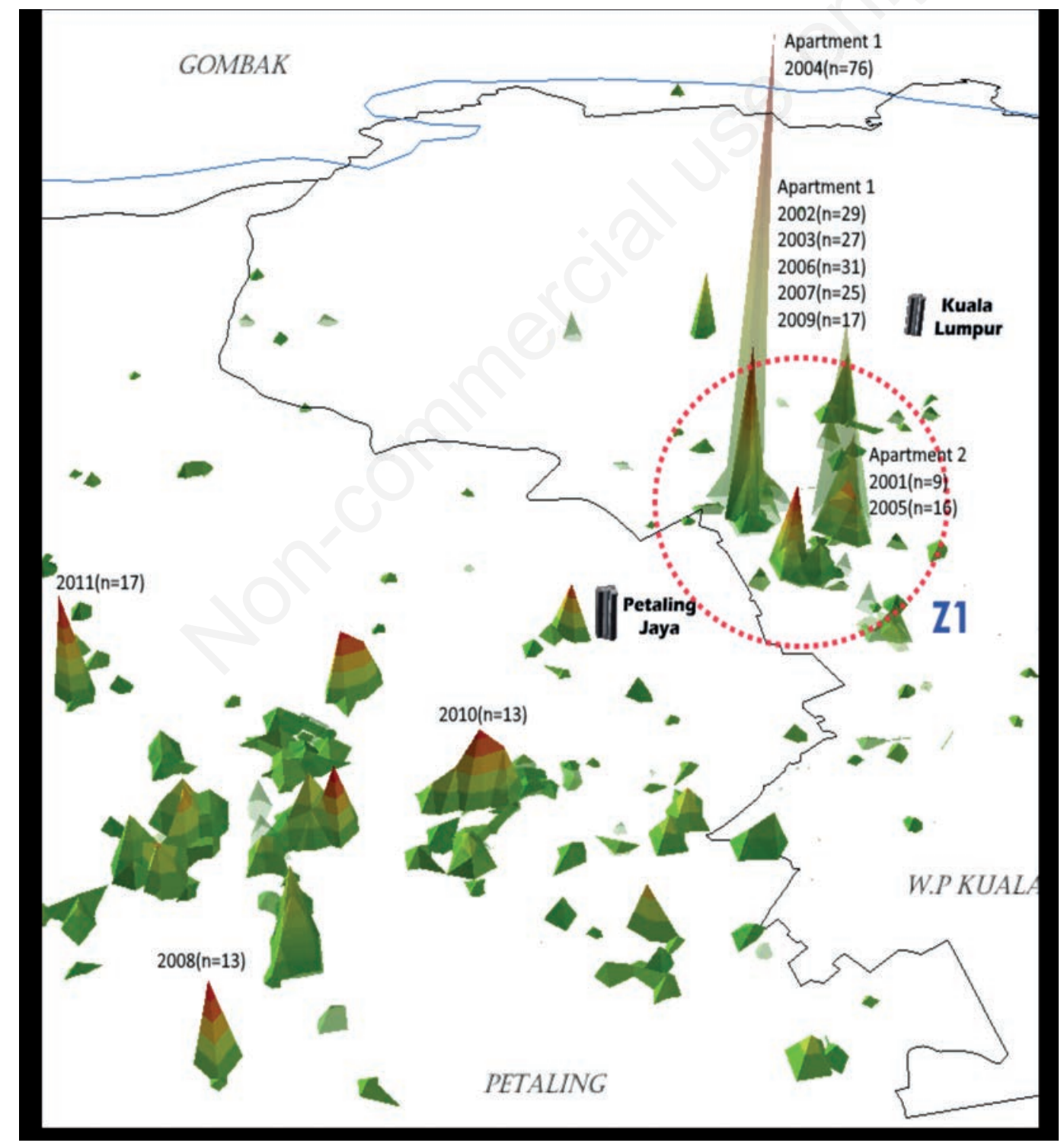

Figure 3. Location and number of the strongest dengue outbreaks in Petaling and Kuala Lumpur. 
(2018) (Yu et al., 2011; Jemal and Al-Thukair, 2018). According to their studies in Taiwan, temperatures higher than $30^{\circ} \mathrm{C}$ can decrease vector growth. However, these findings contrasted with those reported by Marinho (2016) and Liu et al. (2017), who showed that the temperature positively affected the dengue incidence, even when it is above $30^{\circ} \mathrm{C}$. (Marinho et al., 2016; Liu et al., 2017). The increasing temperature accelerates biochemical reactions in the vector increasing viral development as well as vector reproduction, thus increasing dengue transmission risk (Scott and Morrison, 2010; Chan and Johansson, 2012). However, increased temperatures do not only speed up replication and incubation rates but can also negatively impact vector fitness and its capacity to transmit the virus (Lafferty, 2009). Previous observations found a negative correlation between temperature and dengue outbreak during summer, when the increased temperature may hin- der the viral growth (Liu et al., 2020). Other than that, the long school holiday in summer could also contribute to the low dengue incidence rate as most children stay at home. Thus, there is less physical interaction with peers than when at school. Although the exact mechanisms of weather effects on dengue transmission largely remain unclear, we show that that temperature significantly influences the infection rate. Nonetheless, further studies are warranted to better understand the complex relationship between weather variables and dengue transmission.

Dengue has a complex epidemiology as it includes many dynamic transmission interactions between virus, vector and the human host. These factors influence the transmission directly and indirectly, and our study cannot account for all possible variables. Additional data are needed, such as knowledge of virus serotype and genotype, host immune status, vector density, urban water sup-

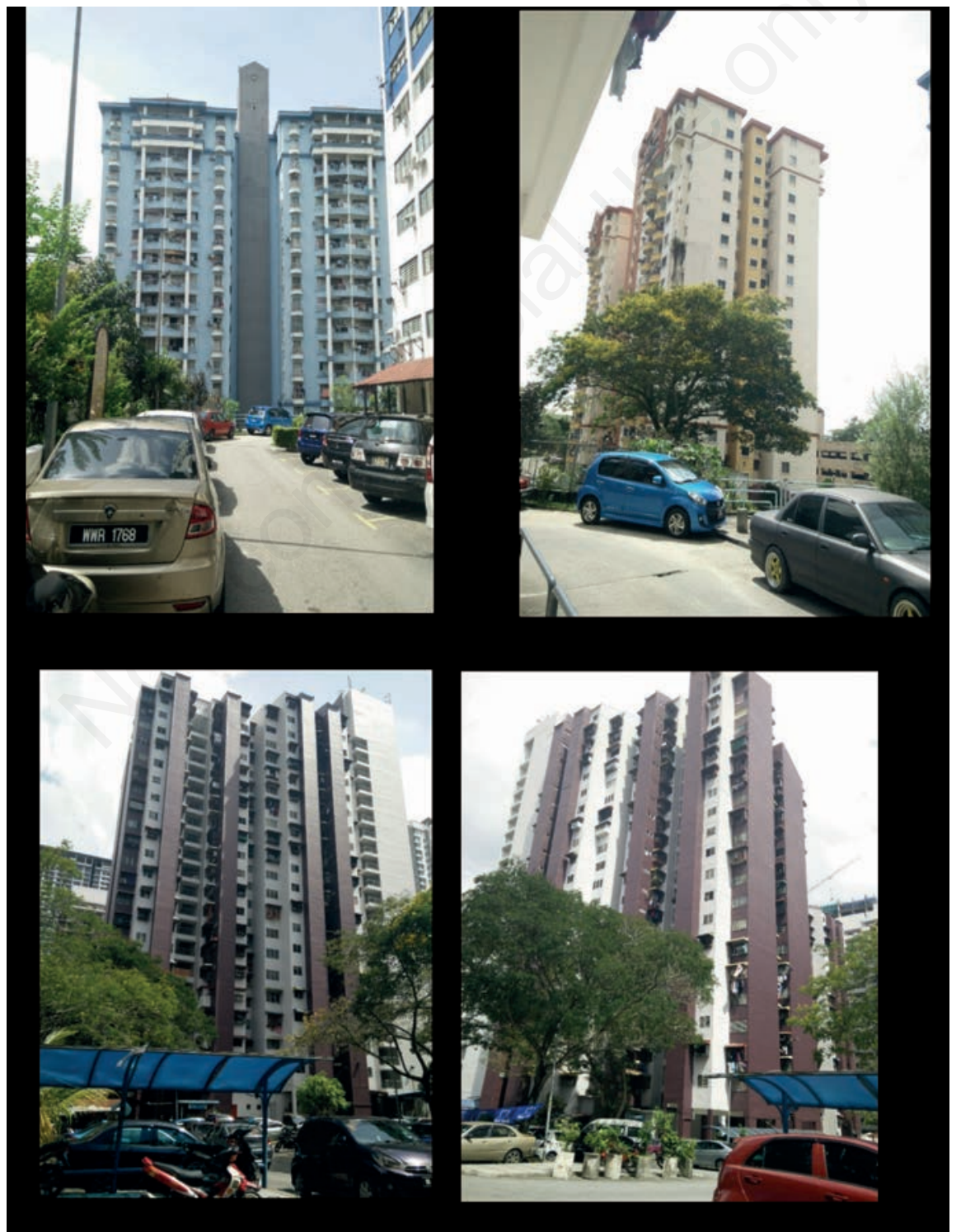

Figure 4. Two high-rise residential apartment buildings in the study areas that had high numbers of dengue outbreaks. Top: Apartment Building 1; Bottom: Apartment Building 2 (for location see Figure 3). 
ply and waste management data. Knowledge and application of this multitude of data should contribute significantly to combating dengue transmission in the future.

One possible limitation lies in the sole use of retrospective data. This is due to the process of construction of GIS data that takes long time as well as technical problems involving the quality of the original dengue data. However, this study has shown a feasible way to utilize better disease surveillance records and environmental information for the study of spatio-temporal disease patterns and environmental risk assessment. The surveillance records of previous years can be studied retrospectively to look for a trend or pattern that can provide advice for future decision-making in disease prevention and control.

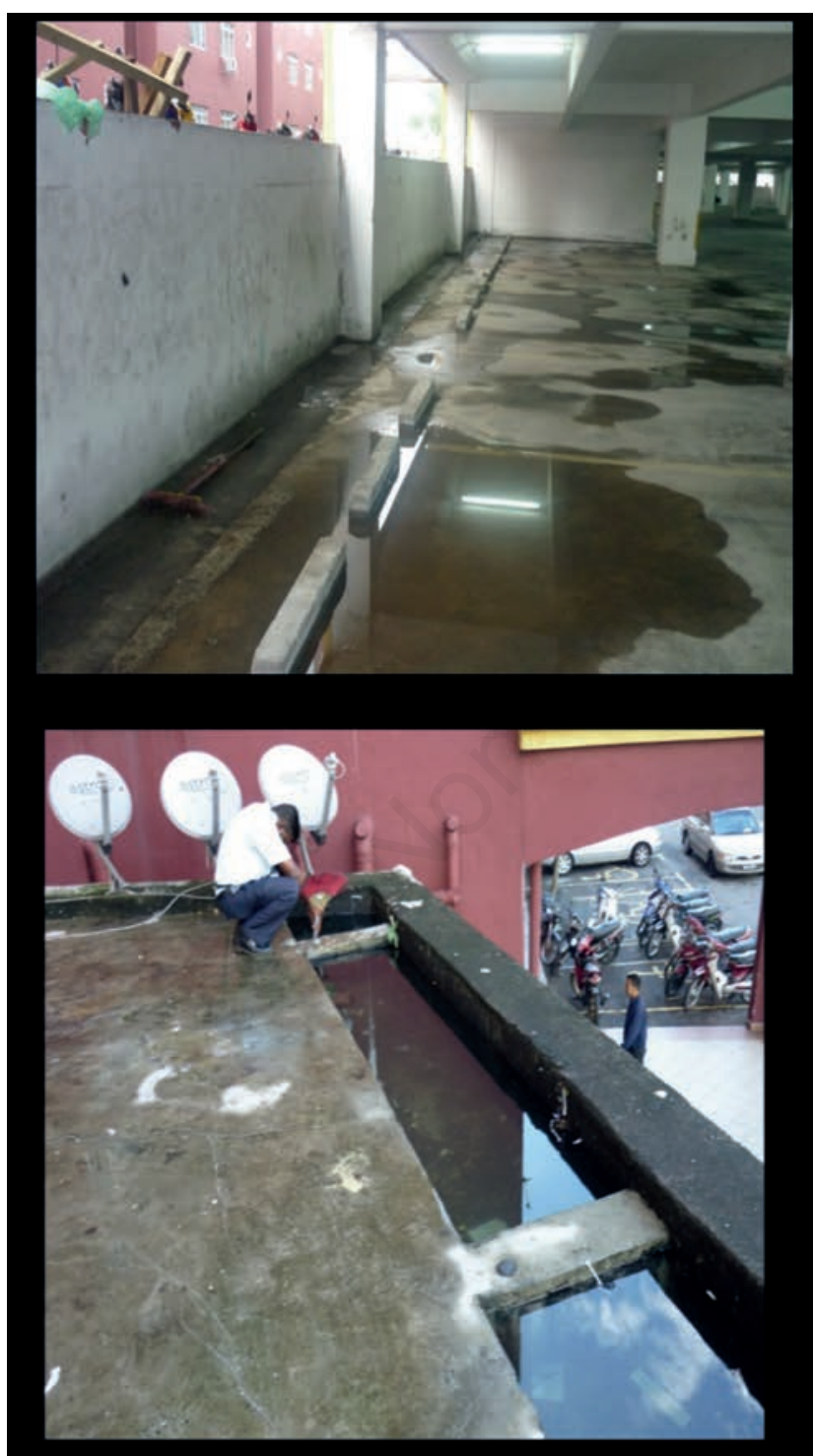

\section{Conclusions}

We conclude that dengue remains a major threat to socio-economic activities and certain geographical conditions promote mosquito reproduction and disease transmission. The geospatial application through quadrant analysis described here identified residential areas with high population densities as the places with the highest number of dengue outbreaks, and that the temperature variable plays a major role for dengue transmission. Since neither drugs nor vaccines are currently available, vector control is the only way to break the transmission cycle. Findings from this study should contribute to the planning of more effective vector control interventions as geospatial applications can support public health departments and agencies in planning control and surveillance.

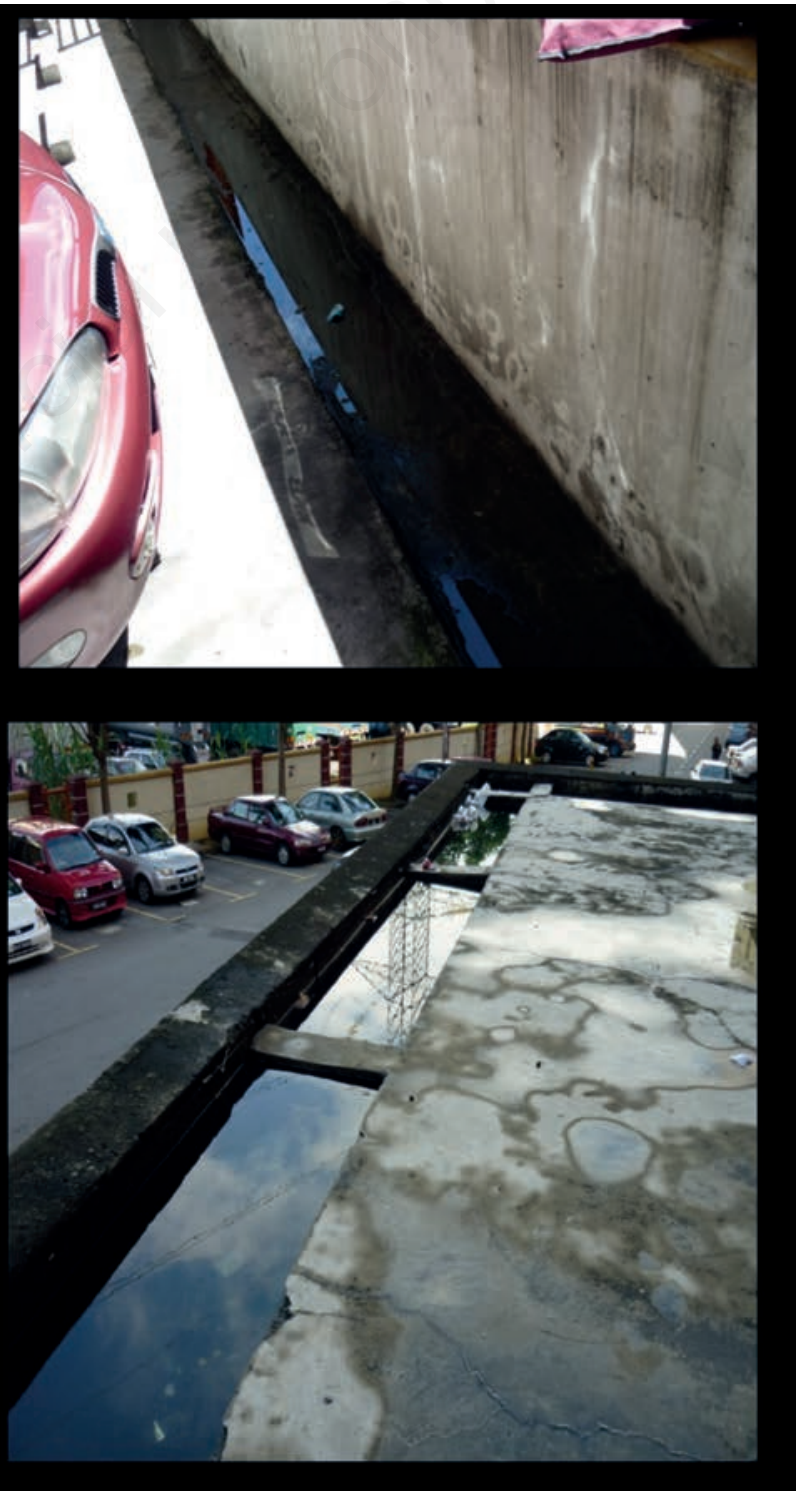

Figure 5. Stagnant water in the high-rise apartment buildings, which provides ideal habitats for Aedes mosquito breeding. Top: Apartment Building 1; Bottom: Apartment Building 2 (for location see Figure 3). 


\section{References}

Aris T, 2019. Prolonged dengue outbreak at a high-rise apartment in Petaling Jaya, Selangor, Malaysia: A case study. Trop Biomed 36:550-8.

Beatty ME, Beutels P, Meltzer MI, Shepard DS, Hombach J, Hutubessy R, 2011. Health economics of dengue: a systematic literature review and expert panel's assessment. Am J Trop Med Hyg 84:473-88.

Braga C, Luna CF, Martelli CM, De Souza WV, Cordeiro MT, Alexander N, 2010. Seroprevalence and risk factors for dengue infection in socio-economically distinct areas of Recife, Brazil. Acta Trop 113:234-40.

Chan M, Johansson MA, 2012. The incubation periods of dengue viruses. PLoS One 7:e50972.

Chang MS, Christophel EM, Gopinath D, Abdur RM, 2011. Challenges and future perspective for dengue vector control in the Western Pacific Region. Western Pac Surveill Response J WPSAR 2:9.

Cheong YL, Leitão PJ, Lakes T, 2014. Assessment of land use factors associated with dengue cases in Malaysia using boosted regression trees. Spat Spatio-temporal Epidemiol 10:75-84.

Crisis Preparedness and Response Centre C, 2020. Dengue in Malaysia. Accessed: 1 Oct 2020.

Dom NC, Ahmad AH, Adawiyah R, Ismail R, 2010. Spatial mapping of temporal risk characteristic of dengue cases in Subang Jaya. Proceedings of the Science and Social Research (CSSR), 2010 International Conference: IEEE, 361-6.

Er A, Rosli M, Asmahani A, Mohamad Naim M, Harsuzilawati M, 2010. Spatial mapping of dengue incidence: a case study in Hulu Langat District, Selangor, Malaysia. Int J Humanit Soc Sci 5:410-4.

Gibbons RV, 2010. Dengue conundrums. Int J Antimicrob Agents 36:S36-S9.

Gubler DJ, 2011. Dengue, urbanization and globalization: the unholy trinity of the 21 st century. Trop Med Health 39:3.

Guzman A, Istúriz RE, 2010. Update on the global spread of dengue. Int J Antimicrob Agents 36:S40-2.

Guzman MG, Halstead SB, Artsob H, Buchy P, Farrar J, Gubler DJ, 2010. Dengue: a continuing global threat. Nat Rev Microbiol 8:S7-16.

Halstead SB, 2007. Dengue. Lancet 370:1644-52.

Hawley WA, Reiter P, Copeland RS, Pumpuni CB, Craig GB, 1987. Aedes albopictus in North America: probable introduction in used tires from northern Asia. Science 236:1114-6.

Hayes JM, García-Rivera E, Flores-Reyna R, Suárez-Rangel G, Rodríguez-Mata T, Coto-Portillo R, 2003. Risk factors for infection during a severe dengue outbreak in El Salvador in 2000. Am J Trop Med Hyg 69:629-33.

Honório NA, Castro MG, Barros FSMD, Magalhães MDaFM, Sabroza PC, 2009. The spatial distribution of Aedes aegypti and Aedes albopictus in a transition zone, Rio de Janeiro, Brazil. Cad Saude Publica 25:1203-14.

Iyagba R, 2005. The menace of sick building; a challenge to all for its prevention and treatment: an inaugural lecture. University of Lagos Press, Lagos, Nigeria.

Jemal Y, Al-Thukair AA, 2018. Combining GIS application and climatic factors for mosquito control in Eastern Province, Saudi Arabia. Saudi J Biol Sci 25:1593-602.

Lafferty KD, 2009. The ecology of climate change and infectious diseases. Ecology 90:888-900.
Lau K, Chen CD, Lee H, Izzul A, Asri-Isa M, Zulfadli M, 2013. Vertical distribution of Aedes mosquitoes in multiple storey buildings in Selangor and Kuala Lumpur, Malaysia. Trop Biomed 30:36-45.

Lawson AB, Williams FL, Williams F, 2001. An introductory guide to disease mapping. Wiley Online Library, New York, NU, USA.

Lee C, Vythilingam I, Chong C-S, Razak MaA, Tan C-H, Liew C, 2013. Gravitraps for management of dengue clusters in Singapore. Am J Trop Med Hyg 88:888.

Lee HL, Chen CD, Masri SM, Chiang YF, Chooi KH, Benjamin S, 2008. Impact of larviciding with a Bacillus thuringiensis israelensis formulation, VectoBac WG, on dengue mosquito vectors in a dengue endemic site in Selangor State, Malaysia. Southeast Asian J Trop Med Public Health 39:601-9.

Liu K, Hou X, Ren Z, Lowe R, Wang Y, Li R, 2020. Climate factors and the East Asian summer monsoon may drive large outbreaks of dengue in China. Environ 183:109190.

Liu Z, Zhang Z, Lai Z, Zhou T, Jia Z, Gu J, 2017. Temperature increase enhances Aedes albopictus competence to transmit dengue virus. Front Microbiol 8:2337.

Lowe R, Bailey TC, Stephenson DB, Graham RJ, Coelho CA, Carvalho MS, 2011. Spatio-temporal modelling of climatesensitive disease risk: Towards an early warning system for dengue in Brazil. Comput Geosci 37:371-81.

Ma S, Ooi EE, Goh KT, 2008. Socioeconomic determinants of dengue incidence in Singapore. Accessed: 1 Oct 2020.

Malaysia MOH, 2009. Pelan Strategik Pencegahan dan Kawalan Denggi 2009-2013. Malaysia Ministry of Health, Kuala Lumpur, Malaysia.

Marinho RA, Beserra EB, Bezerra-Gusmão MA, Porto VDS, Olinda RA, Dos Santos CA, 2016. Effects of temperature on the life cycle, expansion, and dispersion of Aedes aegypti (Diptera: Culicidae) in three cities in Paraiba, Brazil. J Vector Ecol 41:1-10.

Moha A, Maru M, Lika TJ, 2020. Assessment of malaria hazard, vulnerability, and risks in Dire Dawa City Administration of eastern Ethiopia using GIS and remote sensing. Appl 12:15-22.

Mohammed SH, Ahmed MM, Mohammed ZH, Adeboye AJB, Journal BR, 2019. High risk disease mapping and spatial effect of pulmonary tuberculosis in Kerbala, Iraq. Biomed Biotechnol Res J 3:150.

Murugesan B, Karuppannan S, Mengistie AT, Ranganathan M, Gopalakrishnan GJJOGS, 2020. Distribution and Trend Analysis of COVID-19 in India. Geospat Approach 4:1-9.

Nazni W, Lee H, Wan Rozita W, Lian A, Chen C, Azahari A, 2009. Oviposition behaviour of Aedes albopictus in temephos and Bacillus thuringiensis israelensis-treated ovitraps. Accessed: 15 Oct 2020.

Nazri C, Hashim A, Rodziah I, 2009. Distribution pattern of a dengue fever outbreak using GIS. J Environ Health Res 9:89-96.

$\mathrm{Ng} \mathrm{LC}$, 2011. Challenges in dengue surveillance and control. Western Pac Surveill Response J 2:1-3.

Otu A, Ebenso B, Etokidem A, Chukwuekezie O, 2019. Dengue fever - an update review and implications for Nigeria, and similar countries. Afr Health Sci 19:2000-7.

Paz-Soldan VA, Plasai V, Morrison AC, Rios-Lopez EJ, GuedezGonzales S, Grieco JP, 2011. Initial assessment of the acceptability of a Push-Pull Aedes aegypti control strategy in Iquitos, Peru and Kanchanaburi, Thailand. Am J Trop Med Hyg $84: 208$. 
Rodhain F, Rosen L, Gubler D, Kuno G, 1997. Mosquito vectors and dengue virus-vector relationships. In: Dengue and dengue hemorrhagic fever. CAB International, New York, NY, USA, pp 45-60.

Rudnick A, Tan EE, Lucas JK, Omar MB, 1965. Mosquito-borne haemorrhagic fever in Malaya. Br Med J 1:1269-72.

Scott TW, Morrison AC, 2010. Longitudinal field studies will guide a paradigm shift in dengue prevention. Vector Biol Ecol Control 139-61.

Simmons CP, Farrar JJ, Nguyen VV, Wills B, 2012. Dengue. N Engl J Med 366:1423-32.

Skae FM, 1902. Dengue fever in Penang. Br Med J 2:1581-2.

Smith CE, 1957. A localized outbreak of dengue fever in Kuala Lumpur: serological aspects. J Hyg (Lond) 55:207-23.

Thammapalo S, Chongsuwiwatwong V, Geater A, Lim A, Choomalee K, 2005. Socio-demographic and environmental factors associated with Aedes breeding places in Phuket, Thailand. Southeast Asian J Trop Med Public Health 36:426-33.

Wan-Norafikah O, Nazni W, Noramiza S, Shafa'ar-Ko'ohar S, Heah S, Nor-Azlina A, 2012. Distribution of Aedes mosquitoes in three selected localities in Malaysia. Sains Malays 41:1309-13.

Wen T-H, Lin NH, Chao D-Y, Hwang K-P, Kan C-C, Lin KC-M, 2010. Spatial-temporal patterns of dengue in areas at risk of dengue hemorrhagic fever in Kaohsiung, Taiwan, 2002. Int J Infect Dis 14:e334-e43.

Wen TH, Lin NH, Lin CH, King CC, Su MD, 2006. Spatial mapping of temporal risk characteristics to improve environmental health risk identification: A case study of a dengue epidemic in Taiwan. Sci Total Environ 367:631-40.

World Health Organisation W, 2019. Dengue situation update 2019. Accessed: 1 Dec 2020.

World Health Organization W, 2009. Dengue: guidelines for diagnosis, treatment, prevention and control. World Health Organization, Geneva, Switzerland.

Yasuno M, Tonn RJ, 1970. A study of biting habits of Aedes aegypti in Bangkok, Thailand. Bull World Health Organ 43:319.

Yu H-L, Yang S-J, Yen H-J, Christakos G, 2011. A spatio-temporal climate-based model of early dengue fever warning in southern Taiwan. Stoch Environ Res Risk Assess 25:485-94. 